\title{
An Overview of Diverse Techniques on Mobile Charging in the Wireless Rechargeable Networks Yeshwanth Rao Bhandayker ${ }^{1}$
}

${ }^{1}$ Senior Java/J2EE Programmer Analyst, FINANCE: Trading Application Vanguard, Malvern, PA - 19355, USA

\begin{abstract}
Wireless rechargeable sensor networks (WRSNs) have actually become a Traditional Service in order to solve the concern such as network dimension as well as procedure time brought on by typical battery-powered sensor networks. In this paper, the research of wireless energy transfer methods which opened as a brand-new measurement to solve the network lifetime issue exists. Based upon this significant layout restraints on the energy in the sensor network has actually been developed with ideal service. The ideal service is attained in regards to optimizing the billing energy of sensor with rate control in the energy transfer. Despite determining the efficiency proportion of the remedy, its time intricacy, billing proportion was analyzed in each strategy of wireless energy transfer to design a unique service versus the spatial as well as temporal constraints. Additionally, sensor replenishment organizing needs to be automated based upon energy and also navigating of the mobile battery chargers. Ultimately considerable research was likewise continued the speculative results of the conventional formulas in order to evaluate their efficiency versus the network utility rate.
\end{abstract}

Keywords : Wireless Rechargeable Sensor Network, Mobile Charging, Network Utility Maximization.

\section{INTRODUCTION}

Wireless Sensor Connect with minimal energy is significant style restrictions versus the far away information interaction[1]. Wireless energy transfer modern technology is manipulated in the wireless rechargeable sensor network (WRSNs), making it possible for the transmission of electric energy from a battery charger to sensor nodes, leads a brand-new means of renewing the energy or expanding the lifetime of sensor nodes[2]. In many mobile billing situations, the activity of the battery charger is time and also room constricted. In addition, the mobile battery charger can be incorporated with the mobile base terminal to aid relieve network blockage as well as prevent energy locations throughout information collection. When it comes to unidirectional wireless charging, the quantity of energy butted in nodes is defined in regards to the range in between sensor nodes and also the battery charger as well as the period of billing each node. Particularly, the billing power at nodes reduces as the range to the battery charger boosts. It is hence preferable to bill nodes as long as feasible as well as at minimal ranges from the battery charger. Nonetheless, as a result of area variety, the billed energy at various nodes cannot be optimized all at once.

The rate of the battery charger plays an essential function in energy stipulation in WRSNs Because of the non-uniform circulation of sensor nodes; it is non-trivial to constantly establish if the battery charger needs to relocate much faster or slower along the course, in order to take full advantage of the billed energy at nodes. In this paper, a substantial research study is brought in the varied strategy 
related to the node butting in the wireless rechargeable Sensor Network. Based upon this significant layout restraints on the energy in the sensor network has actually been created with ideal service.

The optimum remedy is accomplished in regards to optimizing the billing energy of the sensor via rate control in the energy transfer. Rather than gauging the efficiency proportion of the service as well as its time intricacy, the billing proportion was analyzed in each strategy of wireless energy transfer to design a unique option versus the spatial as well as temporal restrictions. Additionally, sensor replenishment organizing needs to be automated based upon energy and also navigating of the mobile chargers.

\section{REVIEW OF LITERATURES}

The evaluation of the literary works is evaluated in regards to Wireless Energy Transfer as well as billing restrictions in the Wireless Rechargeable sensor networks. Additionally, its significance in the enhancing the Network energy is likewise parallelly examined thoroughly.

\section{A. Collaborative mobile charging for sensor networks}

The joint mobile charging is an energy billing method to Wireless Sensor Network in these mobile chargers is enabled to bill each node in the comparable network geography. The Organizing several mobile chargers are taken a look at carefully which collaboratively charge sensing units, to make the most of the proportion of the quantity of haul energy to overhanging energy, such that every sensor will certainly not lack energy. 2 various restrictions are assessed in which initial think about the consistent situation where all sensing units take in energy at the very same price, an organizing formula, Press Wait, which has actually been shown to be optimum in one-dimensional WSN of unlimited size. Second, in the non-uniform situation, which is assumed to be NP-hard, 2 monitorings from the area and also time facets to get rid of some difficult organizing options exists with a heuristic formula called as ClusterCharging $(\beta)$, which collections sensing units right into teams and also splits an organizing cycle right into billing rounds. Its estimation proportion is likewise offered [5].

\section{B. Data gathering in WRSNs and Joint mobile energy replenishment}

In this Literary works, consistent as well as high reenergizing prices and also energy effective information event from sensing units has actually been supplied by using the movement for joint energy replenishment and also information event[6]. Specifically, a multi-functional mobile entity, called SenCar has actually been used, which offers not just like a mobile information enthusiast to collect information through short-range interaction yet additionally serves as an energy carrier that bills fixed sensing units on its movement by means of wireless energy transmissions. Using SenCar's regulated flexibility, the joint optimization of reliable energy billing as well as high-performance information collections is accomplished with arbitrary geographies. A two-step technique for the joint layout has actually been performed. In the very first step, the places of a part of sensing units are regularly chosen as support factors, where the SenCar will sequentially circulate to bill the sensing units at these areas as well as collect information from close-by sensing units in a multi-hop style. To accomplish a preferable equilibrium in between energy replenishment quantity as well as information event latency, an option formula to look for an optimal 
variety of support factors where sensing units hold the least battery energy was given [6] In the 2nd action, information event efficiency when the SenCar moves amongst these support factors have actually thought about. A dispersed formula to change information prices at which sensing units send out buffered information to the SenCar link organizing and also circulation directing so regarding adjusting to the updated energy restoring condition of sensing units is likewise been examined.

\section{Controlled sink mobility for prolonging wireless sensor networks lifetime}

In this literary works, regulated wheelchair in wireless sensor networks (WSNs) for boosting their life time has actually been assessed i.e., the time period the network has the ability to supply its designated performances. Extra particularly, for WSNs that consist of a lot of statically positioned sensor nodes transferring information to a collection factor (the sink), can be made it possible for by managing the sink motions to acquire impressive life time enhancements.

In order to establish sink activities, a Mixed Integer Linear Programming (MILP) logical version whose option establishes those sink paths that make best use of network life time has actually been made it possible for. By specifying the heuristics for regulated sink motions are been developed as completely dispersed and also local [7] The Greedy Maximum Residual Energy (GMRE) heuristic relocates the sink from its present area to a brand-new area as if attracted towards the location where nodes have the highest possible recurring energy. The relocating sink constantly boosts network life time is been identified from every protocol.

\section{Optimization of Data gathering by dynamic sensing and routing in rechargeable sensor networks}

In this literary works, the energy collected by sensing units ought to be very carefully assigned for information noticing and also information transmission in order to maximize information collecting versus time-varying renewable resource arrival as well as minimal battery ability. Furthermore, the vibrant function of network geography has actually been considered, considering that it can impact the information transmission. In the very first component, a well-balanced energy allowance plan for every sensor to handle its energy usage, which is confirmed to fulfill 4 demands increased by sensible circumstances. After that in the 2nd component, a dispersed noticing price and also directing control (DSR2C) formula to collectively enhance information picking up as well as information transmission, while assuring network justness has actually been talked about. In DSR2C, each sensor can adaptively readjust its transmission energy usage throughout network procedure according for offered energy, and also choose the optimum picking up directing and also the price which can effectively boost information celebration.

\section{OUR PROPOSED MODEL}

The speed control of the mobile chargers taking a trip the 2D Trajectories is maximized making use of the Spatio Temporal Dynamic Routing (STDR) Strategy to Rechargeable Wireless Sensor Network. The Wireless Nodes energies the vibrant node surveillance versions need to collect the information regarding the energy of each node to information tasting price, Node failing and also link failing and so on. Every node shows various efficiency versus the motion of mobile battery charger depends upon the numerous requirements; for this reason, we make use of joint heuristic service to manage to bill activity of the mobile fees based upon the need. The 
recommended formula, as a result, attains greater network energy than existing methods. Additionally, the influence of link/battery ability and also preliminary battery degree on the network energy is additionally explored.

\section{CONCLUSION}

In this paper, a considerable testimonial is performed on the mobile billing control device of Wireless rechargeable sensor networks (WRSNs). The thorough evaluation of those system returns efficient info versus making the most of the network lifetime. Based upon this, ideal remedy in the direction of regulating the node billing can be designed making use of heuristic problem integrating both spatial and also temporal restraints. The problems make the best use of the billing energy of sensor with rate control in the energy transfer. Automating of the sensor replenishment organizing offers network utility rate. Joint optimization of relocating course as well as rate is likewise worth checking out in order to boost the network lifetime as well as energy harvesting.

\section{REFERENCES}

[1]. S. He, J. Chen, F. Jiang, D.K.Y. Yau, G. Xing, and Y. Sun. Energy provisioning in wireless rechargeable sensor networks. IEEE Transactions on Mobile Computing, 12(10):1931-1942, Oct 2013.

[2]. Jiming Chen, Junkun Li, Shibo He, Tian He, Yu $\mathrm{Gu}$, and Youxian Sun. On energy-efficient trap coverage in wireless sensor networks. ACM Trans. Sen. Netw., 10(1):2:1-2:29, December 2013.

[3]. H. Dai, X. Wu, G. Chen, L. Xu, and S. Lin, "Minimizing the number of mobile chargers for large-scale wireless rechargeable sensor networks," Computer Communications, vol. 46, pp. 54-65, 2014.

[4]. S. Zhang, J. Wu, and S. Lu. Collaborative mobile charging for sensor networks. In IEEE MASS, 2012.

[5]. M. Zhao, J. Li, and Y. Yang. A framework of joint mobile energy replenishment and data gathering in wireless rechargeable sensor networks. IEEE Trans. on Mobile Comput., 13(12):2689-2705, Dec 2014.

[6]. E. Melachrinoudis C. Petrioli S. Basagni, A. Carosi and Z. M. Wang. Controlled sink mobility for prolonging wireless sensor networks lifetime. Wireless Networks, 14:831858, 2008.

[7]. Y. Zhang, J. Chen, S. He, "Data gathering optimization by dynamic sensing and routing in rechargeable sensor networks," IEEE/ACM Transactions on Networking,2016.

[8]. S. He, J. Chen, , Y. Sun, D. K. Yau "Cross-layer optimization of correlated data gathering in wireless sensor networks," IEEE Transactions on Mobile Computing, vol. 11, no. 11, pp. 1678-1691, 2012.

[9]. Yeshwanth Rao Bhandayker, "AN OVERVIEW OF THEINTEGRATION OF ALL DATA MINING AT CLOUD-COMPUTING" in "Airo International Research Journal", Volume XVI, June 2018 ISSN : 2320-3714]

[10]. Yeshwanth Rao Bhandayker , “Artificial Intelligence and Big Data for Computer Cyber Security Systems" in "Journal of Advances in Science and Technology”, Vol. 12, Issue No. 24, November-2016 ISSN : 2230-9659]

[11]. Sugandhi Maheshwaram, "A Comprehensive Review on the Implementation of Big Data Solutions" in "International Journal of Information Technology and Management", Vol. XI, Issue No. XVII, November-2016 ISSN : 2249-4510] 
[12]. Sugandhi Maheshwaram , "An Overview of Open Research Issues in Big Data Analytics" in "Journal of Advances in Science and Technology”, Vol. 14, Issue No. 2, September2017 ISSN : 2230-9659]

[13]. Yeshwanth Rao Bhandayker, "Security Mechanisms for Providing Security to the Network" in "International Journal of Information Technology and Management", Vol. 12, Issue No. 1, February-2017, ISSN : 2249-4510]

[14]. Sugandhi Maheshwaram, S. Shoban Babu , "An Overview towards the Techniques of Data Mining" in "RESEARCH REVIEW International Journal of Multidisciplinary", Volume-04, Issue-02, February-2019 ISSN : 2455-3085]

[15]. Yeshwanth Rao Bhandayker, "A Study on the Research Challenges and Trends of Cloud Computing" in "RESEARCH REVIEW International Journal of Multidisciplinary ", Volume-04, Issue-02, February-2019 ISSN : 2455-3085]

[16]. Mounika Reddy, Avula Deepak, Ekkati Kalyani Dharavath, Kranthi Gande, Shoban Sriramoju, "Risk-Aware Response Answer for Mitigating Painter Routing Attacks" in "International Journal of Information Technology and Management", Volume VI, Issue I, Feb 2014 ISSN : 2249-4510 ]

[17]. Sugandhi Maheshwaram, "A Review on Deep Convolutional Neural Network and its Applications" in "International Journal of Advanced Research in Computer and Communication Engineering", Vol. 8, Issue No. 2, February-2019 ISSN : 2278-1021], DOI 10.17148/IJARCCE.2019.8230

\section{Cite this article as :}

Yeshwanth Rao Bhandayker, "An Overview of Diverse Techniques on Mobile Charging in the Wireless Rechargeable Networks", International Journal of Scientific Research in Science and Technology (IJSRST), Online ISSN : 2395-602X, Print ISSN : 2395-6011, Volume 6 Issue 2, pp. 767-771, March-April 2019. Available at doi : https://doi.org/10.32628/IJSRST1962142 Journal URL : http://ijsrst.com/IJSRST1962142 